\title{
多壁碳纳米管固相微萃取纤维制备及其在 海水中多溴联苯测定中的应用
}

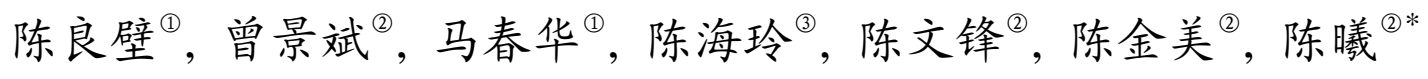

(1) 武夷学院化学与环境工程系, 武夷山 354300;

(2) 厦门大学化学化工学院化学系现代分析科学教育部重点实验室, 厦门 361005;

(3) 厦门华夏职业学院食品药品安全检测中心, 厦门 361005

* 通讯作者, E-mail: xichen@xmu.edu.cn

收稿日期: 2009-07-23 ; 接受日期: 2009-08-04

\begin{abstract}
摘要通过以 Nafion为黏合剂、不锈钢丝为涂层载体, 制备了多壁碳纳米管固相微萃取 纤维. 该纤维的制备方法快速、简便、成本低, 并具有热稳定性好 $\left(300^{\circ} \mathrm{C}\right)$ 、使用寿命长 ( $>100$ 次)、对多溴联苯萃取效率高等特点. 研究优化了影响萃取及分离效率的解吸温度 和时间、萃取时间、摚拌速度、盐度等实验条件, 进行了海水中多溴联苯的测定. 对一溴联苯 的线性范围为 $0.1 \sim 5.0 \mathrm{ng} / \mathrm{mL}$, 而二溴联苯、三溴联苯、四溴联苯和五溴联苯的线性范围均为 $0.01 \sim 5.0 \mathrm{ng} / \mathrm{mL}$. 方法的检测限为 $0.1 \sim 0.8 \mathrm{ng} / \mathrm{L}$. 在优化的条件下分别测定了 0.1 和 $1 \mathrm{ng} / \mathrm{mL}$ 多 溴联苯的海水加标样品, 回收率在 $91.1 \%$ 107.3\%之间, 相对标准偏差小于 $12 \%$. 该方法分 析时间短、灵敏度高、操作简便, 适用于水样中多溴联苯的痕量分析.
\end{abstract} 关键词 多壁碳纳米管 固相微萃取 多溴联苯

\section{1 引言}

多溴联苯 $(\mathrm{PBBs})$ 具有优异的阻燃性，被广泛用 于纺织, 电子电器和塑料制品等高聚物的阻燃处理 ${ }^{[1]}$. 然而, 由于其具有亲脂性, 难降解性和生物富集性, 对人体健康有较大的危害. 因此, 欧盟和联合国环境 规划署均颁布相关法令严格限制 PBBs 的使用 ${ }^{[2]}$. 为 了保护自然环境和消费者的安全, 迫切需要建立快 速、灵敏的PBBs测定方法.

目前PBBs的测定主要采用色相色谱 (GC), 气质 联用 (GC/MS) 和液质联用 (LC/MS) 分析技术 ${ }^{[2 \sim 4]}$. 前处理主要采用索氏提取法, 超声波萃取法, 固相萃

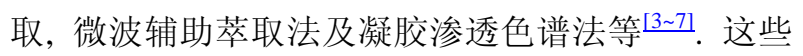
前处理方法都存在一些不足之处,或需要大量的有机 溶剂,或运行成本高、操作复杂,操作时间长等,这些不
利因素制约了上述方法在痕量分析中的应用. 固相 微萃取 (SPME) 是 20 世纪 90 年代由 Pawliszyn研究 小组 ${ }^{[8]}$ 首创, 集萃样、萃取、浓缩、进样于一体的无 溶剂样品预处理技术. 该技术的工作原理是基于分 析待测物在纤维涂层的吸附平衡, 而达到萃取目的. 因此, 选择与制备对目标物具有较强亲和性的纤维 涂层对提高测定方法的灵敏度和检测限至关重要. 自 1991 年Iijima ${ }^{[9]}$ 发现碳纳米管 (CNT) 以来, 其独 特的一维管状分子结构和奇特的物理化学特征引起 科学家的广泛关注. CNT具有很大的比表面积和较高 的热稳定性, 对有机物, 特别是含苯环结构的有机物 有着很强的吸附能力, 因此在化学分离, 如气相色谱 固定相、固相萃取柱制备得到广泛应用 ${ }^{[10]}$. 然而, 目 前国内外应用CNT制备固相微萃取涂层的研究报道 
较少 $[11$ 13] . 通常 CNT 先被分散于有机溶剂或有机黏 合剂中, 形成CNT的悬浮液, 再将空白石英纤维反复 浸渍到该悬浮液, 高温老化, 待达到一定厚度后, 得 到CNT-SPME纤维。该制备过程较为繁琐，并且方法 存在一些不足: 一方面有机溶剂的挥发易造成CNT 的脱落, 缩短纤维的使用寿命; 另一方面有机黏合剂 高温下的流失可能会形成干扰目标物定性的鬼峰.

Nafion 是一种全氟磺酸离子交换剂, 具有耐热、 耐酸碱等稳定的化学性能. 国外曾有研究小组直接 将其作为 SPME 涂层萃取极性化合物, 取得良好效果. 研究工作以 Nafion 为黏合剂、不锈钢为涂层载体, 采 用物理浸渍法制备了多壁碳纳米管 (MWCNTs) SPME 纤维, 并与气相色谱 (GC) 联用进行了海水中 PBBs 的测定. 对影响 SPME 萃取效率的萃取时间、 温度、搅拌速度以及离子强度等影响因素进行了详细 的考察和优化, 建立了海水中 PBBs 的 SPME-GC 分 析方法.

\section{2 实验部分}

\section{1 仪器及工作条件}

日本岛津 GC-2010型气相色谱仪 (配 ECD 检测 器), RTX-1毛细管柱 $(30 \mathrm{~m} \times 0.25 \mathrm{~mm}$ i.d. $\times 0.25 \mu \mathrm{m}$ film), 实验选用色谱控制方式: 恒压 $135.8 \mathrm{kPa}$, 无分 流进样 $5 \mathrm{~min}$, 进样口温度: $280^{\circ} \mathrm{C}$, 检测器温度: $300^{\circ} \mathrm{C}$, 载气和尾吹气：高纯氮 (含量 $\geqslant 99.999 \%$ ), 总流量 $19.5 \mathrm{~mL} / \mathrm{min}$, 尾吹流量: $60 \mathrm{~mL} / \mathrm{min}$, 柱温程序: $120^{\circ} \mathrm{C}$ (恒温 $2 \mathrm{~min}$ ) $-20^{\circ} \mathrm{C} / \mathrm{min}$ 升温至 $240^{\circ} \mathrm{C}$ (恒温 $2 \mathrm{~min}$ ) $-10^{\circ} \mathrm{C} / \mathrm{min}$ 升温至 $280^{\circ} \mathrm{C}$ (恒温 $2 \mathrm{~min}$ ). 固相微萃取时 间: $25 \mathrm{~min}$, 进样口解吸附时间: $5 \mathrm{~min}$.

实验选用具有不同极性的商品化 SPME萃取纤 维, $75 \mu \mathrm{m}$ 碳分子笁/聚二甲基硅氧烷 (Car/PDMS), $85 \mu \mathrm{m}$ 聚丙烯酸酯 (PA) 和65 $\mu \mathrm{m}$ 聚二甲基硅氧烷/ 聚二乙烯基苯 (PDMS /DVB) (Supelco, Bellefonte, PA, USA), 进行对比实验. SPME 纤维涂层的表面结构及 涂层厚度使用扫描电镜Hitachi S4800 (Tokyo, Japan) 进行表征.

\section{2 试剂}

多澳联苯标准品: 一溴联苯(Mono-BB), 二溴联 苯 (Di-BB), 三溴联苯 (Tri-BB), 四澳联苯 (Tetra-BB),
五溴联苯 (Penta-BB) 均购自 ChemService公司 (PA, USA). 丙酮为农药残留级 (TEDIA 公司, Ohio, USA). 每种多溴联苯标准品用丙酩逐级稀释成浓度为 $1 \mu \mathrm{g} / \mathrm{mL}$ 的储备溶液. SPME实验用的混合标准溶液均用水稀释 至所需浓度, 实验用水为经Milli-Q纯水器 (MILLIPORE 公司, Bedford, MA, USA) 净化的超纯水. 5\% Nafion 溶液购自 Aldrich 公司 (Milwaukee, WI, USA). 多壁 碳纳米管 (长度: 1 2 $\mu \mathrm{m}$; 直径: $<10 \mathrm{~nm}$ )购自深圳纳米 港有限公司. 不锈钢丝(直径: $0.15 \mathrm{~mm}$ )购自安亭微量 进样器厂.

\subsection{MWCNTs SPME 纤维的制备}

实验采用本研究小组自制的SPME手柄 ${ }^{[14]}$. 将不 锈钢丝用丙酮、乙醇以及二次去离子水依次清洗后晾 干，待用. 取 5\%Nafion溶液 $200 \mu \mathrm{L}$ 和MWCNTs $50 \mathrm{mg}$ 分别置于 $250 \mu \mathrm{L}$ 微量离心管中, 两者在离心管中的 高度均约为 $1.5 \mathrm{~cm}$. 将不锈钢丝插入Nafion溶液中浸 泡 $2 \mathrm{~min}$, 取出后速插入MWCNTs中, 取出后在 $160^{\circ} \mathrm{C}$ 烘箱中加热 $30 \mathrm{~min}$, 重复上述操作一次后, 制得 MWCNTs SPME纤维涂层. 其表面结构如图 1 所示, MWCNTs均匀地固定在不锈钢纤维表面, 涂层厚度 约为 $25 \mu \mathrm{m}$. 使用时, 将MWCNTs SPME纤维在 $280^{\circ} \mathrm{C}$ 老化, 至无杂峰出现.

\section{3 结果与讨论}

\subsection{MWCNTs SPME 纤维的热稳定性}

为考察 MWCNTS SPME 纤维的热稳定性, 纤维 分别在 $240,260,280,300,320^{\circ} \mathrm{C}$ 下老化 $2 \mathrm{~h}$, 比较其 萃取效率的变化. 实验结果表明, 经 $240^{\circ} \mathrm{C} 300^{\circ} \mathrm{C}$ 老 化, 纤维的萃取效率未有明显的改变. 当老化温度升 至 $320^{\circ} \mathrm{C}$ 后，经多次重复使用后，涂层粉末易脱落而 使萃取效率有所下降. 因此, 该纤维的最高使用温度 可达 $300^{\circ} \mathrm{C}$. 实验所用的纤维在 GC 进样口 $280^{\circ} \mathrm{C}$ 的条 件下使用次数超过 100 次, 未见萃取效率明显变化.

\subsection{MWCNTs SPME 纤维萃取能力}

为了考察 MWCNTS SPME 纤维的萃取能力, 在 相同的实验条件下, 将该纤维与不锈钢丝、Nafion 纤 维进行萃取能力比较. 结果表明 (图2), 空白不锈钢 丝对目标物几乎不具有萃取能力. Nafion 纤维虽然对 

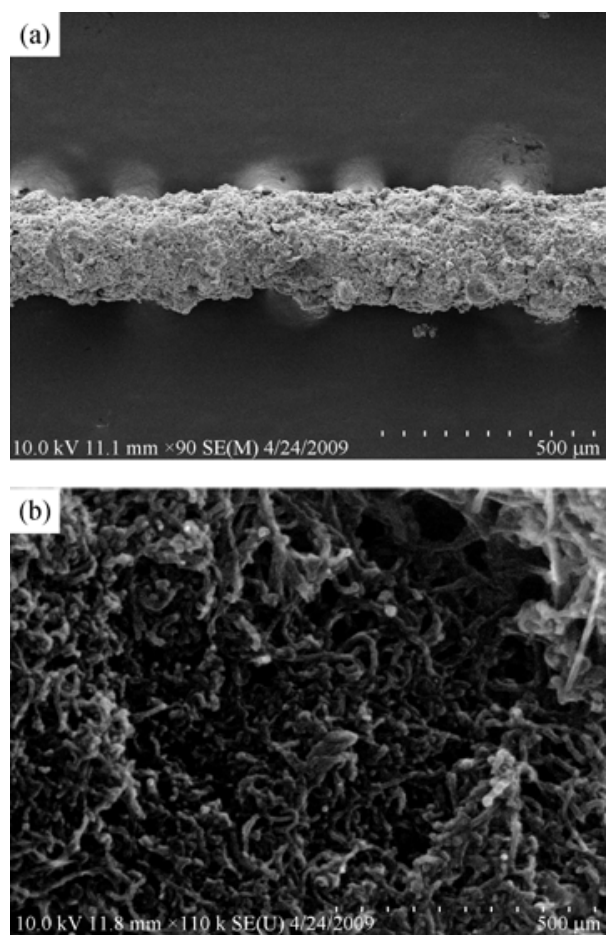

图 1 MWCNTs SPME 纤维的 SEM 图

(a)放大倍数 90 倍; (b) 放大倍数 11000 倍

目标物具备一定的吸附能力, 但是萃取效率显著小 于 MWCNTs SPME 纤维. 实验结果说明, 被 Nafion 固定于不锈钢纤维表面的 MWCNTs 对提高纤维的萃 取能力, 起重要的作用.

为了更加准确和有效的衡量 MWCNTs 纤维的萃 取能力, 实验选用几种不同极性的商品化 SPME 萃取 纤维 $85 \mu \mathrm{m}$ PA, $75 \mu \mathrm{m}$ CAR/PDMS 和 $65 \mu \mathrm{m}$ PDMS/ DVB), 进行了它们萃取能力的比较. 由图 3 可知, MWCNTs SPME纤维对 Mono-BB、Tetra-BB, Penta$\mathrm{BB}$ 的萃取量大于所有商品化萃取纤维. Tri-BB的萃 取只有 $75 \mu \mathrm{m}$ CAR/PDMS 纤维与 MWCNTs SPME 纤 维相当; 对 Di-BB 的萃取, 只有 $85 \mu \mathrm{m}$ PA 的纤维萃 取能力大于 MWCNTs 纤维. 整体上, MWCNTs SPME 纤维对 5 种目标物的萃取能力大于商品化 SPME 萃取 纤维. 由于 MWCNTs 纤维的涂层厚度远小于所选用 的商品化 SPME 纤维厚度, 说明 MWCNTs 纤维对 $\mathrm{PBBs}$ 的吸附能力明显大于商品化 SPME 纤维. MWCNTs SPME 纤维对 PBBs 有良好的萃取能力, 主 要是由于 MWCNTs 有着类似于石墨的六边形网络所

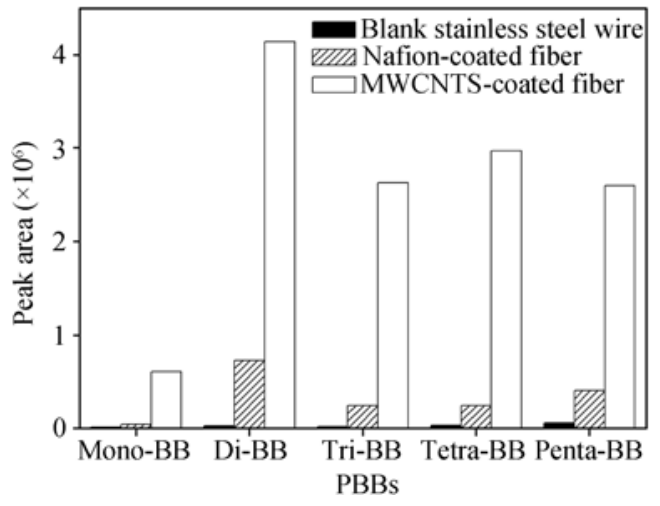

图 2 MWCNTs SPME 纤维与空白不锈钢纤维、Nafion 纤 维萃取效率对比

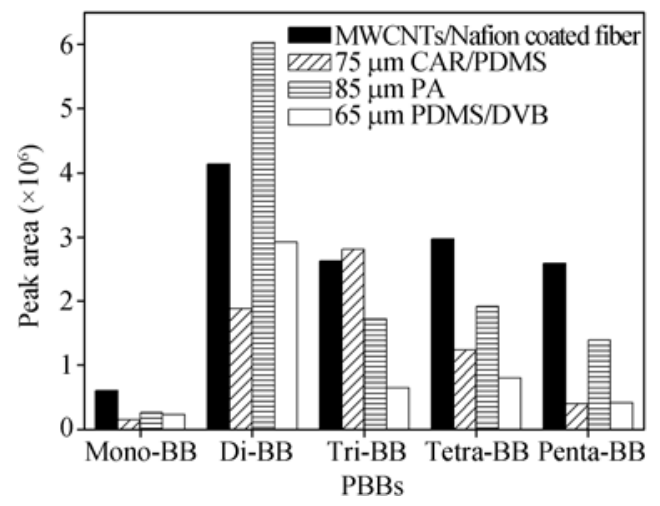

图 3 MWCNTs SPME 纤维与商品化纤维萃取效率对比

组成的管状纳米中空结构, 具有较大的比表面积和 疏水的石墨表面，能有效的吸附 PBBs. 同时, MWCNTs 存在的 $\pi-\pi$ 分子结构可以和多溴联苯的联 苯环结构通过大 $\pi$ 键形成很强相互作用, 从而进一步 促进了 PBBs 在 MWCNTs 纤维上的吸附.

\subsection{SPME 主要参数的优化}

(1) 解吸温度, 解吸时间的优化. 在 SPME-GC 应用中, 解吸温度太低, 被萃取物质的不能有效解吸, 滞留物将影响后续分析; 温度太高, 则可能使待测物 质分解, 同时也可能会影响萃取纤维的使用寿命. 实 验探讨进样口温度为 $220^{\circ} \mathrm{C}, 240^{\circ} \mathrm{C}, 260^{\circ} \mathrm{C}, 280^{\circ} \mathrm{C}$ 和 $300^{\circ} \mathrm{C}$ 时 $\mathrm{PBBs}$ 解吸情况, 结果表明, 升高温度有利于 待测物质有效脱附, 在 $220 \sim 280^{\circ} \mathrm{C}$, 各物质的峰面积 随解吸温度升高而增大. 但在 $300^{\circ} \mathrm{C}$ 时, 出现了一些 杂峰, 说明某些多溴联苯在 $300^{\circ} \mathrm{C}$ 时发生了分解. 因 此后续实验中选用的解吸温度为 $280^{\circ} \mathrm{C}$. 为了考察解 
吸时间对待测物脱附的影响, 实验分别选择了解吸 时间为 2, 5, 8和 $11 \mathrm{~min}$, 结果表明解吸时间为 2 8 $\mathrm{min}$ 时, 峰面积随解吸时间增而增加, 而在 $11 \mathrm{~min}$ 时, 解 吸峰面积与 $8 \mathrm{~min}$ 相近, 说明在 $8 \mathrm{~min}$ 时, 待测物达到 了解吸平衡状态, 为提高本实验工作效率, 选择 $8 \mathrm{~min}$ 为 SPME 的解吸时间.

(2) 萃取时间的影响. 为了考察萃取时间对萃取 效率的影响, 实验设计了萃取 5, 10, 15, 20, 25 和 $30 \mathrm{~min}$ 系列实验. 图 4 的实验结果表明, 随着萃取时间的增 加, 固相涂层对溶液中待测物的吸附量也增大, 至萃 取时间 $25 \mathrm{~min}$ 时多数待测物萃取量基本达到最大值 并开始趋于平衡. 根据Louch ${ }^{[15]}$ 提出的直接浸入固相 微萃取(DI-SPME) 方法的数字模型以及 $\mathrm{Ai}^{[16]}$ 提出的 非平衡固相微萃取的动态吸附模型, 在其他条件固 定时, 涂层对待测物的萃取量正比于溶液中待测物 的起始浓度, 在非平衡状态下，可进行定量分析. 因此 综合考虑萃取量和工作效率, 选择萃取时间为 $15 \mathrm{~min}$.

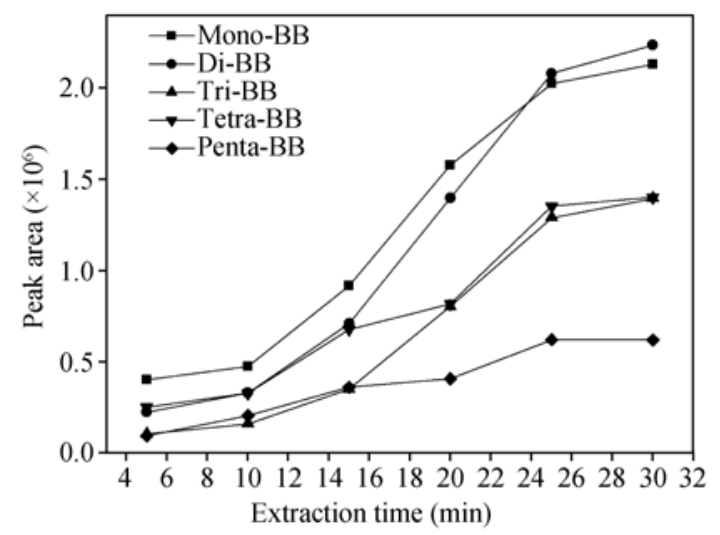

图 4 萃取时间对萃取效率的影响

(3) 搅拌速度的影响. SPME 本质上是固-液间 萃取过程. 在萃取过程中, 存在于溶液中的待测物质 通过固-液间扩散层向固相涂层表面扩散，进而发生 吸附的过程. 因此, 适当的搅拌有利于溶液本体中的 待测物分子通过扩散层向固体涂层表面运动而提高萃 取效率. 实验考察了 0 750 r/min 搅拌速度时MWCNTs SPME 纤维对 PBBs 的萃取效率. 如图 5 所示的实验 结果表明, 随搅拌速度增加待测物色谱峰面积增大, 至 $600 \mathrm{r} / \mathrm{min}$ 时, 各待测物色谱峰面积达最大值, 当转速 达750 r/min 时, 容易导致搅拌子弹跳和液面中心下凹 现象, 影响了萃取的正常进行, 使萃取效率下降, 同时

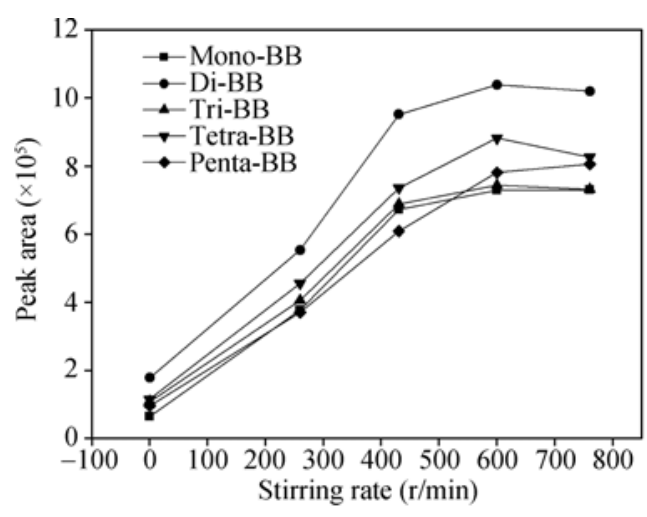

图 5 搅拌速度对萃取效率的影响

也降低了萃取重现性. 实验选择搅拌速度为 $600 \mathrm{r} / \mathrm{min}$.

(4) 盐度的影响。在 SPME操作中, 一般认为, 在水溶液中加入无机盐能增大溶液中的离子强度并 降低有机物在基质中的溶解度, 从而提高分配系数, 增加待测组分的萃取量, 进而提高分析的灵敏度. 但 通过无机盐的方法来提高萃取效率，并非适用于任 何液体样品及任何待测组分. 徐盈 [17]等研究结果表 明, 在进行 HS-SPME-GE 测定模拟水中的多氯联苯 (PCBs) 时, 随着 $\mathrm{NaCl}$ 浓度的增加, PCBs的萃取量反 而减少, Shu等 ${ }^{[18]}$ 在研究中也发现了这一现象.

实验设计了含 $\mathrm{NaCl} 0 \%, 2 \%, 4 \%, 8 \%, 16 \%(\mathrm{~W} / \mathrm{W})$ 的 PBBs 标准溶液体系, 进行盐度影响探讨. 实验结 果表明, 盐度影响较为复杂, 其总体趋势为: 当浓度 为 $2 \% \sim 4 \%$ 时, 萃取量没有明显的变化, $8 \%$ 时萃取量 略有增大，当 $\mathrm{NaCl}$ 浓度继续增大至 $16 \%$ 时，萃取效 率又开始下降上述现象的产生为盐析效应与盐溶作 用共同影响的结果. 因此在后续实验中, 采用不加无 机盐的纯水体系.

\subsection{SPME-GC 分析的线性范围、检测限和精密度}

在优化的实验条件下，用 4 根同批制备的 MWCNTs SPME 纤维进行重现性实验. 实验表明, 所 制得的 MWCNTS SPME 纤维间重现性良好, 相对标 准偏差 $<11.4 \%$. 配制 $0.01 \sim 5.0 \mathrm{ng} / \mathrm{mL}$ 含 5 种 PBBs 的 系列混标溶液, 进行方法的线性范围、检测限和精密 度的测定. 实验结果见表 1 .

由表 1 可知, 建立的SPME-GC法对一溴联苯的 线性范围为 $0.1 ~ 5.0 \mathrm{ng} / \mathrm{mL}$, 而二溴联苯, 三溴联苯, 四溴联苯和五溴联苯的线性范围均为 $0.01 \sim 5.0 \mathrm{ng} / \mathrm{mL}$, 
相关系数 $\mathrm{R}^{2}$ 在 0.99 以上, 相对标准偏差在 $4.6 \%$ 12.5\% 之间，所建立的方法完全满足外标法分析待测物的 条件. 在所设定的GC条件下，由于MWCNTs SPME 纤维对PBBs的强吸附性以及 ECD检测器对PBBs的灵 敏响应, 方法具有较高的灵敏度和较低的检测限. 5 种PBBs的检测限为 $0.1 \sim 0.8 \mathrm{ng} / \mathrm{L}$. 其检测限低于相关 文献报道的SPME-GC分析环境水样中PBBs的检测限, 3.6 8.6 ng/ $\mathrm{L}^{[19]}, 0.14 \sim 6.74 \mathrm{ng} / \mathrm{L}^{[20]}$. 所建立的 SPME-GC-ECD法具有分析速度快(<35 min)、无需任 何有机溶剂、灵敏度高等特点, 在PBBs残留分析领域 应具有较好的应用前景.

表 1 SPME 法的线性范围、检测限, 相关系数和精密度

\begin{tabular}{lcccc}
\hline \begin{tabular}{c} 
样品 \\
\multicolumn{1}{|}{ 称 }
\end{tabular} & $\begin{array}{c}\text { 检测限 } \\
(\mathrm{ng} / \mathrm{L})\end{array}$ & $\begin{array}{c}\text { 线性范围 } \\
(\mathrm{ng} / \mathrm{mL})\end{array}$ & 相关系数 $R^{2}$ & $\begin{array}{c}\mathrm{RSD} \% \\
(n=5)\end{array}$ \\
\hline Mono-BB & 0.8 & $0.1 \sim 5.0$ & 0.9984 & 9.5 \\
Di-BB & 0.1 & $0.01 \sim 5.0$ & 0.9974 & 5.7 \\
Tri-BB & 0.1 & $0.01 \sim 5.0$ & 0.9974 & 4.6 \\
Tetra-BB & 0.3 & $0.01 \sim 5.0$ & 0.9951 & 6.5 \\
Penta-BB & 0.4 & $0.01 \sim 5.0$ & 0.9938 & 12.5 \\
\hline
\end{tabular}

\section{5 实际样品分析和方法回收率}

在优化的 SPME 和 GC-ECD 的实验条件下, 分析 了厦门观音山海域的海水样品, 结果未检出多溴联 苯目标物(表 2). 在海水样中分别加入标准溶液, 制 得 PBBs 浓度为 0.1 和 $1.0 \mathrm{ng} / \mathrm{mL}$ 的加标样品, 进行加 标回收测试(图6). 分析结果表明, 方法的回收率在 91.1\% 107.3\%之间, 相对标准偏差小于 $12 \%$, 满足痕 量分析的要求.

\section{4 结论}

研究采用不锈钢丝为涂层载体、Nafion 为黏合剂, 制备了 MWCNTs SPME 纤维, 并将该纤维与 GC 联
表 2 SPME-GC 方法测定实际海水结果

\begin{tabular}{|c|c|c|c|c|c|}
\hline 样品名称 & $\begin{array}{l}\text { 测定值 } \\
\text { (ng/mL) }\end{array}$ & $\begin{array}{c}\text { 回收率 }{ }^{a)} \\
(\%)\end{array}$ & $\begin{array}{c}\text { RSD } \\
(n=3, \%)\end{array}$ & $\begin{array}{c}\text { 回收率 }{ }^{b)} \\
(\%)\end{array}$ & $\begin{array}{c}\text { RSD } \\
(n=3, \%)\end{array}$ \\
\hline Mono-BB & $\mathrm{ND}^{\mathrm{c})}$ & 104.4 & 3.1 & 97.3 & 8.2 \\
\hline Di-BB & ND & 107.2 & 5.5 & 91.1 & 8.8 \\
\hline Tri-BB & ND & 104.5 & 9.6 & 92.5 & 10.0 \\
\hline Tetra-BB & ND & 96.8 & 10.0 & 96.2 & 10.7 \\
\hline Penta-BB & ND & 107.3 & 12.0 & 96.3 & 3.0 \\
\hline
\end{tabular}

a) 加标 $0.1 \mathrm{ng} / \mathrm{mL}$; b) 加标 $1 \mathrm{ng} / \mathrm{mL}$;) 未检出

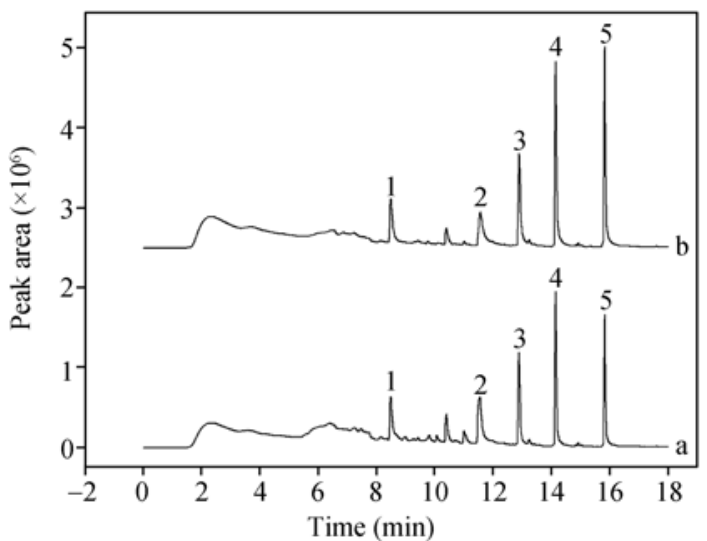

图 6 SPME-GC 方法在优化条件下分析海水(a)和纯水 (b) 中的加标样品

加标浓度: $1 \mathrm{ng} / \mathrm{mL}$; 峰序号: 1. Mono-BB, 2. Di-BB, Tri-BB, Tetra-BB, Penta-BB

用进行了海水中 PBBs 的测定. 实验结果表明，与商 品化纤维相比, MWCNTs 纤维对 PBBs 具有很强的萃 取能力, 能在较小厚度下 $(25 \mu \mathrm{m})$ 实现对 $\mathrm{PBBs}$ 的有效 萃取. 此外, 该纤维具有较好的热稳定性 $\left(300{ }^{\circ} \mathrm{C}\right)$ 、机 械强度和较长的使用寿命 (>100 次), 能用于实际分析. 建立的 SPME-GC 法对 PBBs 检测的线性范围宽、检 测限低 (ppf 级)、重现性好, 有望在 PBBs 快速检测 中得到应用.

致谢本工作得到了福建省自然科学基金(批准号: 2006J0393)、福建省科技计划重点项目(批准号: 2007Y0032, 2009Y0045)和福建省南平市科技计划项目(批准号: NP2006Z1-1)资助，特此一并致谢.

\section{参考文献}

1 萧达辉, 肖前, 周明辉, 刘莹峰, 翟翠萍, 郑建国。红外光谱法定性篎选电气产品中多溴联苯和多溴联苯醚. 中国塑料,2005, 19(12): $74-78$

2 罗峰. 气相色谱-质谱测定塑胶中多溴联苯和多溴联苯醚。光谱实验室, 2008, 25(1): 23-27

3 郡玉婉, 郡超英, 张玩。气相色谱法测试纺织品中多溴联苯阻燃剂. 印染, 2008, 19: 30-34

4 王成云, 杨左军, 张伟亚. 微波辅助萃取-高效液相色谱法测定塑料中的澳系阻燃剂. 塑料助剂, 2006, 56(2): 39-43 
5 范成英, 谢卢明. 微波萃取和气相色谱-质谱测定塑料中的多溴联苯. 化工生产技术, 2008, 15(5): 46-49

6 陈社军,麦碧娴,曾永平,罗孝俊,向同寿,傅家谟,盛国英. 沉积物中多溴联苯醚的测定. 环境科学, 2005, 24(4): 474-477

7 周相娟, 赵玉琪, 常宇文, 李伟, 许华. 凝胶渗透色谱净化 GC-MS 测定食品包装印刷油墨中多澳联苯及多溴联苯醚. 现代 仪器, 2006, 6: 28-31

8 Arthur C L, Pawliszyn J. Solid phase microextraction with thermal desorption using fused silica optical fibers. Anal Chem, 1990, 62: 2145-2148

9 Iijima S. Helical microtubules of graphitic carbon. Nature, 1991, 354: 56-58[DOI]

10 Pyrzynska K. Carbon Nanotubes as a New Solid-Phase Extraction Material for Removal and Enrichment of Organic Pollutants in Water. Sep Puri Rev, 2008, 37(4): 372-389[DOI]

11 Wang J X, Jiang D Q, Gu Z Y, Yan X P. Multiwalled carbon nanotubes coated fibers for solid-phase microextraction of polybrominated diphenyl ethers in water and milk samples before gas chromatography with electron-capture detection. J Chromatogr A, 2006, 1137: 8 - 14[DOI]

12 Lv J X, Liu J F, Wei Y, Jiang K L, Fan S S, Liu J Y, Jiang G B. Preparation of single - walled carbon nanotube fiber coating for solid — phase microextraction of organochlorine pesticides in lake water and wastewater. J Sep Sci, 2007, 30: 2138-2143[DOI]

13 田孟鬼, 冯喜兰. 山药中有机氯杀虫剂的自制碳纳米管探头顶空固相微萃取/气相色谱分析. 分析测试学报, 2008, 27(8): 825一 829

14 Zeng J B, Yu B B, Chen W F, Lin Z J, Zhang L M, Lin Z Q, Chen X, Wang X R. Application of ceramic/carbon composite as a novel coating for solid-phase microextraction. J Chromatogr A, 2008, 1188: 26-33[DOI]

15 Louch D, Motlagh S, Pawliszyn J. Extraction dynamics of organic compounds from water using liquid-coated fused silica fibres. Anal Chem. 1992, 64: 1187-1199[DOI]

16 Ai J. Solid phase microextraction for quantitative analysis in nonequilibrium situations. Anal Chem, 1997, 69: 1230-1236[DOI]

17 赵高峰, 徐盈, 惠阳, 李秀娟, 曾昭㝐. 杯[4]芳烃顶空固相微萃取-气相色谱法快速分析环境样品中的多氯联苯. 分析科学 学报, 2004, 20(6): 561-564

18 Shu Y Y, Wang S S, Tardif M, Huang Y P. Analysis of polychlorinated biphenyls in aqueous samples by microwave-assisted headspace solid-phase microextraction. J Chromatogr A, 2003, 1008: 1-12 [DOI]

19 Wang J X, Jiang D Q, Gu Z Y, Yan X P. Multiwalled carbon nanotubes coated fibers for solid-phase microextraction of polybrominated diphenyl ethers in water and milk samples before gas chromatography with electron-capture detection. J Chromatogr A, 2006, 1137: 8-14[DOI]

20 Basheer C, Vetrichelvan M, Valiyaveettil S, Lee H K. On-site polymer-coated hollow fiber membrane microextraction and gas chromatography - mass spectrometry of polychlorinated biphenyls and polybrominated diphenyl ethers. Chromatography A, 2007, 1139: 157-164 [DOI]

\title{
Preparation of multi-walled carbon nanotubes coated solid-phase microextraction fiber and its application on the analysis of polybromobiphenyls in seawater
}

\author{
CHEN LiangBi ${ }^{1}$, ZENG JingBin ${ }^{2}$, MA ChunHua ${ }^{1}$, CHEN HaiLing ${ }^{3}$, CHEN WenFeng ${ }^{2}$, CHEN JinMei ${ }^{2}$, \\ CHEN Xi ${ }^{2 * *}$
}

\footnotetext{
${ }^{1}$ Department of Chemistry and Environment Engineering, Wuyi University, Fujian, 354300, China

${ }^{2}$ Department of Chemistry and the Key Laboratory of Analytical Sciences of the Ministry of Education, College of Chemistry and Chemical Engineering, Xiamen University, Xiamen 361005, China

${ }^{3}$ Testing Center for Food and Drug Safety, Huaxia Vocational College, Xiamen 361024, China
}

\begin{abstract}
In this study, we proposed an approach using Nafion as a binder to immobilize multi-walled carbon nanotubes (MWCNTs) on a stainless steel wire base to prepare MWCNTs-coated fiber. The preparation method is fast, simple and low-cost. The MWCNTs-coated fiber shows high affinity towards polybromobiphenyls (PBBs), and mechanical and thermal stability. The experimental parameters related to extraction efficiency including desorption temperature and time, extraction time, stirring rate, salt concentration were investigated and optimized. The linearity was found to be from 0.1 to $5.0 \mathrm{ng} / \mathrm{mL}$ for monobromobiphenyl (mono-BB), and 0.01 to $5.0 \mathrm{ng} / \mathrm{mL}$ for dibromobiphenyl (Di-BB), tribromobiphenyl (Tri-BB), tetrabromobiphenyl (Tetra-BB) and pentabromobiphenyl (Penta-BB). The detection limits of these PBBs were between 0.1 and $0.8 \mathrm{ng} / \mathrm{L}$. The recovery of the PBBs spiked in sea water at $0.1 \mathrm{ng} / \mathrm{mL}$ and $1 \mathrm{ng} / \mathrm{mL}$ ranged from $91.1 \%$ to $107.3 \%$, and the relative standard deviations were less than $12 \%$. The proposed method which is sensitive, fast and easy to operate could be applied to the determination of trace-level PBBs in water samples.
\end{abstract}

Keywords: multi-walled carbon nanotubes (MWCNTs), solid-phase microextraction (SPME), polybromobiphenyls (PBBs) 